\title{
Predictors of Severe or Moderate Coronary Artery Disease in Asymptomatic Individuals with Extremely Low Coronary Calcium Scores
}

\author{
Hyung-Bok Park ${ }^{1 *}$, Hyeonju Jeong ${ }^{2 *}$, ji Hyun Lee ${ }^{2}$, Yongsung Suh ${ }^{2}$, \\ Eui-Seock Hwang', Yun-Hyeong $\mathrm{Cho}^{2}$, and Deok-Kyu Cho ${ }^{2}$ \\ ${ }^{1}$ Division of Cardiology, Department of Internal Medicine, International St. Mary's Hospital, Catholic Kwandong University College of Medicine, \\ Incheon; \\ ${ }^{2}$ Division of Cardiology, Department of Internal Medicine, Myongji Hospital, Hanyang University College of Medicine, Goyang, Korea.
}

Purpose: To evaluate predictors of severe or moderate coronary artery disease (CAD) in individuals with zero or very low $(<10)$ coronary artery calcium (CAC) scores.

Materials and Methods: The 1175 asymptomatic persons with zero or very low $(<10)$ CAC scores were analyzed for CAD stenosis using coronary computed tomography angiography. Moderate and severe CADs were defined as having more than $50 \%$ and more than $70 \%$ stenosis in any of the major coronary arteries, respectively. Age, gender, body mass index, hypertension, type II diabetes, dyslipidemia, lipid profile, creatinine, and smoking status were evaluated as predictors for moderate and severe CAD.

Results: In the study population, moderate and severe CADs were found in $7.5 \%$ and $3.3 \%$, respectively. Among evaluated risk factors, age [odds ratio (OR) 1.04, 95\% confidence interval (CI) 1.02-1.07, $p<0.001$ ], current smoking status (OR 3.12, 95\% CI 1.82$5.34, p<0.001$ ), and CAC 1-9 (OR 1.80, 95\% CI 1.08-3.00, $p=0.024)$ were significantly associated with moderate CAD. Meanwhile, age (OR 1.05, 95\% CI 1.02-1.08, $p=0.003$ ), low high density lipoprotein (HDL) (OR 0.96, 95\% CI 0.93-0.99, $p=0.003$ ), and current smoking status (OR 2.34, 95\% CI 1.14-5.30, $p=0.022$ ) were found to be significantly associated with severe CAD. Improvement of discrimination power for predicting severe CAD was observed when smoking and HDL cholesterol were serially added into the age model.

Conclusion: Smoking showed significant correlations with moderate or severe CAD, and low HDL cholesterol also proved to be a predictor of severe CAD in asymptomatic individuals with extremely low CAC scores.

Key Words: Asymptomatic condition, coronary artery disease, calcium, computed tomography angiography

\section{INTRODUCTION}

Silent ischemia occurs in $20-40 \%$ of patients with stable and

Received: December 24, 2018 Revised: May 16, 2019

Accepted: May 28, 2019

Corresponding author: Deok-Kyu Cho, MD, Division of Cardiology, Department of Internal Medicine, Myongji Hospital, Hanyang University College of Medicine, 55 Hwasu-ro 14beon-gil, Deogyang-gu, Goyang 10475, Korea. Tel: 82-31-810-5114, Fax: 82-31-969-0500, E-mail: chodk123@paran.com

*Hyung-Bok Park and Hyeonju Jeong contributed equally to this work.

- The authors have no potential conflicts of interest to disclose

(C) Copyright: Yonsei University College of Medicine 2019

This is an Open Access article distributed under the terms of the Creative Commons Attribution Non-Commercial License (https://creativecommons.org/licenses/ by-nc/4.0) which permits unrestricted non-commercial use, distribution, and reproduction in any medium, provided the original work is properly cited. unstable coronary syndromes. Unfortunately, for some patients, their first clinical manifestation is when they go into sudden cardiac arrest. Coronary artery calcium (CAC) score has emerged as a robust predictor of future coronary events in these asymptomatic primary prevention populations. ${ }^{1}$ This noninvasive technique has proven to be a reliable tool for estimating overall coronary plaque burden ${ }^{2}$ and to generally correlate well with the severity of coronary artery stenosis (CAD) ${ }^{2,3}$ However, in some cases, obstructive or even severe $\mathrm{CAD}$ has been detected in patients with zero or very low $(<10)$ calcium scores. ${ }^{4,5}$ These false negative patients should be carefully monitored and may need prompt intervention to prevent the progression of acute coronary syndrome. We aimed to evaluate clinical key predictors of severe $\mathrm{CAD}$ in this population and sought to de- 
velop better prediction models for the detection of severe CAD combined with clinical risk factors.

\section{MATERIALS AND METHODS}

\section{Study population and data collection}

The institutional review board of Myongii Hospital approved this study and waived the requirement for informed patient consent (IRB number: MJH-16-098). In this retrospective and observational study, from April 2011 to April 2016, we consecutively enrolled 3022 asymptomatic subjects who underwent coronary computed tomography angiography (CTA) with coronary calcium scan at the same time during health check-ups or outpatient clinics in Myongji Hospital. Among those populations, 1231 subjects with CAC scores of zero or less than 10 were enrolled. Males or females were excluded from the study if they were younger than 20 years or older than 80 years or had previous history of $\mathrm{CAD}$, such as myocardial ischemia/infarction, percutaneous coronary intervention, or coronary artery bypass graft surgery. Poor quality CTA images were also excluded ( $\mathrm{n}=55)$. Hence, a total of 1176 subjects were analyzed for CAD using CTA. Clinical risk factors, such as age, gender, body mass index (BMI), hypertension (HTN), type II diabetes mellitus (DM), dyslipidemia, lipid profile, and smoking status, were evaluated as predictors for severe CAD.

\section{Measurement of CAC and CTA}

For coronary calcium scan and CTA, we used a 64 multi-detector computed tomography scanner (Lightspeed Volume CT, GE Healthcare, Milwaukee, WI, USA). One hour prior to the CT scan, we administered $100 \mathrm{mg}$ of atenolol to patients who had heart rates of more than 65 beats/min. Immediately before scanning, 0.6-mg sublingual nitroglycerin was given to all patients. Coronary calcium scan was performed before CTA scanning. The calcium scan parameters were as follows: prospective electrocardiogram-triggered at $70 \%$ of the R-R interval, $2.5-\mathrm{mm}$ slice thickness, a gantry rotation time of $350 \mathrm{~ms}$, a tube voltage of $120 \mathrm{kVp}$, and a tube current of $200-250 \mathrm{~mA}$ (depending on the patient's BMI). CAC score was calculated according to the Agatston method. ${ }^{6}$ The analysis was performed using a commercially available external workstation (Advantage Windows, version 4.2, GE Healthcare, Milwaukee, WI, USA), as well as CAC scoring software (Smartscore 3.5, GE Healthcare, Milwaukee, WI, USA). Moderate and severe CAD were defined as having more than $50 \%$ or more than $70 \%$ stenosis in any of the major coronary arteries, respectively.

\section{Statistical analysis}

Data are reported as mean \pm standard deviation for continuous variables and as counts with proportions (\%) for categorical variables. Continuous variables were compared using an unpaired t-test for normally distributed variables or the Mann-
Whitney U test for non-normally distributed variables. Categorical variables were examined by Pearson's chi-square or Fisher's exact test. Global chi-square analyses utilized logistic regressions and likelihood ratios tests. Univariable and multivariable odds ratios (ORs) estimates with $95 \%$ confidence intervals (95\% CIs) were analyzed to evaluate predictors of moderate and severe $\mathrm{CAD}$. Among univariate analysis, significant variables ( $p$ value $<0.05$ ) were tested in multivariate analysis, except age and gender. Ten-fold cross-validated estimates of the area under the receiver operating characteristic curve (AUC) models were employed to evaluate the discrimination of severe CAD. The estimates were using the method proposed by DeLong, et al. ${ }^{7}$ Statistical analyses were performed using MedCalc software (version 17.6; MedCalc Software, Mariakerke, Belgium).

\section{RESULTS}

Among the 1176 subjects, $47.2 \%$ ( $\mathrm{n}=555$ ) were male, with a mean age of $56.5 \pm 12.3$. The mean CAC was $0.8 \pm 2.1$, and zero CAC was observed in $82.7 \%(n=972)$. Moderate $(\geq 50 \%)$ CAD was present in $7.5 \%(\mathrm{n}=88)$ and severe $(\geq 70 \%) \mathrm{CAD}$ in $3.3 \%$ $(\mathrm{n}=39)$ of the total population on CTA. Moderate (14.7\% vs. $6.0 \%, p<0.001)$ and severe $\mathrm{CAD}(6.4 \%$ vs. $2.7 \%, p=0.014)$ were found to be two- to three-fold higher in the non-zero (1 to 9) CAC group than in the zero CAC group (Fig. 1). We found that $37.3 \%(\mathrm{n}=439)$ of the total population had HTN, $12.1 \%(\mathrm{n}=142)$ had DM, and 41.9\% ( $\mathrm{n}=493$ ) presented with dyslipidemia. Statin use status was recorded in $30.0 \%(\mathrm{n}=459)$, and $20.0 \%(\mathrm{n}=$ 235) were current smokers.

\section{Moderate stenosis ( $\geq 50 \%$ ) CAD by CTA}

When divided into two groups, the group with $\geq 50 \%$ stenosis by CTA (the obstructive CAD group) was older $(61.6 \pm 10.7 \mathrm{vs}$. $56.1 \pm 12.3, p<0.001)$, was male predominant $(62.5 \%$ vs. $46.0 \%$, $p=0.004)$, had more DM (21.6\% vs. $11.3 \%, p=0.007)$, had lower high density lipoprotein (HDL) $(47.0 \pm 14.4$ vs. $52.1 \pm 14.4, p=$ $0.002)$, and had fewer subjects with zero CAC $(34.1 \%$ vs. $16.0 \%, p<0.001)$, compared to the non-obstructive CAD group (Table 1). In univariate analysis, age (OR 1.04, 95\% CI 1.02$1.06, p<0.001$ ), male (OR 1.96, 1.25-3.07), DM (OR 2.16, 1.263.71, $p=0.005$ ), low HDL cholesterol (OR 0.97, 0.96-0.99, $p=$ 0.002 ), current smoking status (OR 3.43, 2.19-5.39, $p<0.001$ ), and non-zero CAC (OR 2.72, 1.70-4.35, $p<0.001$ ) were associated with obstructive $\mathrm{CAD}$ (Table 2). In multivariate analysis, age (OR 1.04, 95\% CI 1.02-1.07, $p<0.001$ ), current smoking status (OR 3.12, 1.82-5.34, $p<0.001$ ), and non-zero CAC (OR $1.80,1.08-3.00, p=0.024)$ were significantly associated with obstructive CAD (Table 2).

\section{Severe stenosis ( $\geq 70 \%$ ) CAD by CTA}

The severe CAD group had a stronger likelihood of being old- 

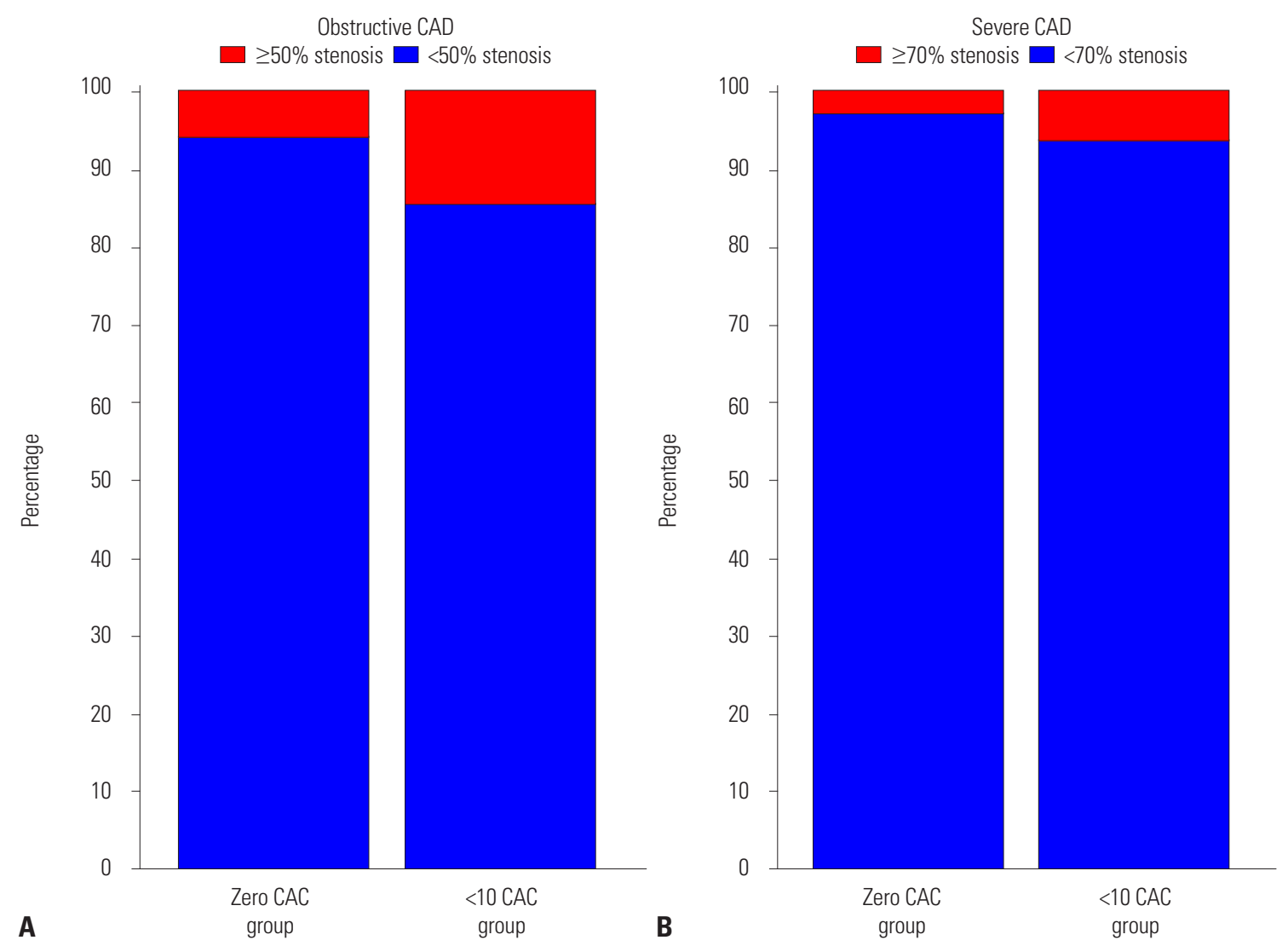

Fig. 1. Prevalence of moderate $C A D(A)$ and severe $C A D(B)$ between the less than $10 C A C$ score group and zero $C A C$ score group. $C A D$, coronary artery disease; CAC, coronary artery calcium.

Table 1. Baseline Patient Characteristics according to CAD Severity

\begin{tabular}{|c|c|c|c|c|c|c|}
\hline & \multicolumn{6}{|c|}{$C A C \leq 10(n=1176)$} \\
\hline & $\begin{array}{c}\geq 50 \% \text { stenosis } \\
(n=88,7.5 \%)\end{array}$ & $\begin{array}{c}<50 \% \text { stenosis } \\
\text { (n=1088, } 92.5 \%)\end{array}$ & $p$ value & $\begin{array}{c}\geq 70 \% \text { stenosis } \\
(n=39,3.3 \%)\end{array}$ & $\begin{array}{c}<70 \% \text { stenosis } \\
\text { (n=1137, } 96.7 \%)\end{array}$ & $p$ value \\
\hline Age (yr) & $61.6 \pm 10.7$ & $56.1 \pm 12.3$ & $<0.001$ & $62.4 \pm 11.2$ & $56.3 \pm 12.3$ & 0.002 \\
\hline Male $(n, \%)$ & $55(62.5)$ & $500(46.0)$ & 0.004 & $24(61.5)$ & $531(46.7)$ & 0.10 \\
\hline BMI & $24.3 \pm 3.3$ & $24.8 \pm 3.7$ & 0.22 & $23.7 \pm 3.2$ & $24.8 \pm 3.7$ & 0.056 \\
\hline $\mathrm{DM}(\mathrm{n}, \%)$ & $19(21.6)$ & $123(11.3)$ & 0.007 & $9(23.1)$ & $133(11.7)$ & 0.058 \\
\hline $\operatorname{HTN}(n, \%)$ & $39(44.3)$ & $400(36.8)$ & 0.20 & $14(35.9)$ & $425(37.4)$ & 0.98 \\
\hline Dyslipidemia (n, \%) & $32(36.4)$ & $461(42.4)$ & 0.32 & $11(28.2)$ & $482(42.4)$ & 0.11 \\
\hline Statin use (n, \%) & $31(35.3)$ & 428 (39.3) & 0.52 & $11(28.2)$ & 448 (39.4) & 0.21 \\
\hline LDL cholesterol (mg/dL) & $104.3 \pm 37.3$ & $109.7 \pm 35.2$ & 0.18 & $105.2 \pm 41.9$ & $109.4 \pm 35.2$ & 0.47 \\
\hline HDL cholesterol (mg/dL) & $47.0 \pm 14.4$ & $52.1 \pm 14.4$ & 0.002 & $42.9 \pm 9.2$ & $52.0 \pm 14.5$ & $<0.001$ \\
\hline $\mathrm{TG}$ (mg/dL) & $145.1 \pm 85.6$ & $141.7 \pm 114.4$ & 0.79 & $144.4 \pm 68.5$ & $141.9 \pm 113.6$ & 0.89 \\
\hline Creatinine (mg/dL) & $1.0 \pm 0.3$ & $1.0 \pm 0.7$ & 0.27 & $1.1 \pm 0.3$ & $1.0 \pm 0.7$ & 0.25 \\
\hline Current smoking $(\mathrm{n}, \%)$ & 38 (43.2) & $197(18.1)$ & $<0.001$ & $16(41.0)$ & $219(19.3)$ & 0.002 \\
\hline CAC score 1-9 (n, \%) & 30 (34.1) & $174(16.0)$ & $<0.001$ & 13 (33.3) & 191 (16.8) & 0.014 \\
\hline LM disease & 2 & - & - & 0 & - & - \\
\hline CAD 1VD & 53 & - & - & 23 & - & - \\
\hline CAD 2VD & 31 & - & - & 12 & - & - \\
\hline CAD 3VD & 4 & - & - & 4 & - & - \\
\hline $\mathrm{PCl}$ & 23 & - & - & 22 & - & - \\
\hline
\end{tabular}

CAD, coronary artery disease; CAC, coronary artery calcium; BMI, body mass index; DM, diabetes mellitus; HTN, hypertension; LDL, low density lipoprotein; HDL, high density lipoprotein; TG, triglyceride; LM, left main; VD, vessel disease; PCl, percutaneous coronary intervention.

Values are presented as mean \pm standard or $\mathrm{n}(\%)$ deviation unless otherwise indicated. 
er (62.4 \pm 11.2 vs. $56.3 \pm 12.3, p=0.002)$ with lower HDL (42.9 \pm 9.2 vs. $52.0 \pm 14.5, p<0.001$ ) than the non-severe CAD group (Table 1). Univariate analysis showed age (OR 1.04, 95\% CI 1.01-1.07, $p=0.003$ ), DM (OR 2.26, 1.05-4.87, $p=0.037$ ), low HDL cholesterol (OR 0.95, 0.92-0.97, $p<0.001$ ), current smoking status (OR 2.92, 95\% CI 1.51-5.61, $p=0.001$ ), and non-zero CAC (1-9) score (OR 2.48, 95\% CI 1.25-4.91, $p=0.009$ ) to be significantly associated with severe CAD (Table 3 ). However, only age (OR 1.05, 95\% CI 1.02-1.08, $p=0.003$ ), low HDL (OR 0.96, 95\% CI 0.93$0.99, p=0.003$ ), and current smoking status (OR 2.45, 95\% CI 1.14-5.30, $p=0.022$ ) were found to be significantly associated with severe CAD in multivariate analysis (Table 3). Improve- ment of discrimination power for predicting severe CAD was observed when smoking and HDL cholesterol were serially added into the age model: the AUC value was higher in the "Age+Smoking+HDL cholesterol" model when compared to the "Age+Smoking" model (AUC 0.77, 95\% CI 0.74-0.80 vs. AUC 0.70, 95\% CI 0.67-0.73, $p=0.020$ ) (Fig. 2).

\section{Zero CAC analysis}

Among the 972 people in the zero CAC group, $6.0 \%(n=58)$ had obstructive $\mathrm{CAD}$ and $2.7 \%(\mathrm{n}=26)$ had severe $\mathrm{CAD}$ (Fig. 1, Tables 4 and 5). Age, males, DM, low HDL cholesterol, and current smoking status were associated with severe CAD in uni-

Table 2. Univariate and Multivariate Aanalysis for Detecting Moderate ( $\geq 50 \%$ stenosis) CAD

\begin{tabular}{|c|c|c|c|c|c|c|}
\hline & \multicolumn{6}{|c|}{$C A C \leq 10(n=1176)$} \\
\hline & \multicolumn{3}{|c|}{ Univariate } & \multicolumn{3}{|c|}{ Multivariate } \\
\hline & Moderate CAD (odds ratio) & $95 \% \mathrm{Cl}$ & $p$ value & Moderate CAD (odds ratio) & $95 \% \mathrm{Cl}$ & $p$ value \\
\hline Age & 1.04 & $1.02-1.06$ & $<0.001$ & 1.04 & $1.02-1.07$ & $<0.001$ \\
\hline Male & 1.96 & $1.25-3.07$ & 0.003 & 1.67 & $0.96-2.91$ & 0.072 \\
\hline BMl & 0.96 & $0.90-1.02$ & 0.22 & - & - & - \\
\hline DM & 2.16 & $1.26-3.71$ & 0.005 & 1.24 & $0.69-2.24$ & 0.48 \\
\hline HTN & 1.37 & $0.88-2.12$ & 0.16 & - & - & - \\
\hline Dyslipidemia & 0.78 & $0.50-1.22$ & 0.27 & - & - & - \\
\hline Statin use & 0.84 & $0.53-1.32$ & 0.45 & - & - & - \\
\hline LDL cholesterol & 1.00 & $0.99-1.00$ & 0.18 & - & - & - \\
\hline HDL cholesterol & 0.97 & $0.96-0.99$ & 0.002 & 0.99 & $0.97-1.01$ & 0.30 \\
\hline TG & 1.00 & 0.99-1.00 & 0.79 & - & - & - \\
\hline Creatinine & 1.12 & $0.90-1.40$ & 0.30 & - & - & - \\
\hline Current smoking & 3.43 & 2.19-5.39 & $<0.001$ & 3.12 & $1.82-5.34$ & $<0.001$ \\
\hline CAC score 1-9 & 2.72 & $1.70-4.35$ & $<0.001$ & 1.80 & $1.08-3.00$ & 0.024 \\
\hline
\end{tabular}

CAD, coronary artery disease; CAC, coronary artery calcium; Cl, confidence interval; BMI, body mass index; DM, diabetes mellitus; HTN, hypertension; LDL, low density lipoprotein; HDL, high density lipoprotein; TG, triglyceride.

Table 3. Univariate and Multivariate Analysis for Detecting Severe ( $\geq 70 \%$ Stenosis) CAD

\begin{tabular}{|c|c|c|c|c|c|c|}
\hline & \multicolumn{6}{|c|}{$C A C \leq 10(n=1176)$} \\
\hline & \multicolumn{3}{|c|}{ Univariate } & \multicolumn{3}{|c|}{ Multivariate } \\
\hline & Severe CAD (odds ratio) & $95 \% \mathrm{CI}$ & $p$ value & Severe CAD (odds ratio) & $95 \% \mathrm{Cl}$ & $p$ value \\
\hline Age & 1.04 & $1.01-1.07$ & 0.003 & 1.05 & $1.02-1.08$ & 0.003 \\
\hline Male & 1.83 & $0.95-3.52$ & 0.07 & 1.50 & $0.68-3.32$ & 0.32 \\
\hline BMI & 0.91 & $0.82-1.00$ & 0.054 & - & - & - \\
\hline DM & 2.26 & $1.05-4.87$ & 0.037 & 1.24 & $0.55-2.78$ & 0.60 \\
\hline HTN & 0.94 & $0.48-1.82$ & 0.85 & - & - & - \\
\hline Dyslipidemia & 0.53 & $0.26-1.08$ & 0.082 & - & - & - \\
\hline Statin use & 0.60 & $0.30-1.23$ & 0.163 & - & - & - \\
\hline LDL cholesterol & 1.00 & 0.99-1.01 & 0.47 & - & - & - \\
\hline HDL cholesterol & 0.95 & $0.92-0.97$ & $<0.001$ & 0.96 & $0.93-0.99$ & 0.003 \\
\hline TG & 1.00 & $1.00-1.00$ & 0.89 & - & - & - \\
\hline Creatinine & 1.15 & $0.89-1.50$ & 0.29 & - & - & - \\
\hline Current smoking & 2.92 & $1.51-5.61$ & 0.001 & 2.45 & $1.14-5.30$ & 0.022 \\
\hline CAC score 1-9 & 2.48 & $1.25-4.91$ & 0.009 & 1.50 & $0.73-3.11$ & 0.27 \\
\hline
\end{tabular}

CAD, coronary artery disease; $C A C$, coronary artery calcium; Cl, confidence interval; BMI, body mass index; DM, diabetes mellitus; HTN, hypertension; LDL, low density lipoprotein; HDL, high density lipoprotein; TG, triglyceride. 
variate analysis (Table 5). When adjusted for these variables, age (OR 1.06, 95\% CI 1.02-1.10, $p=0.002$ ), low HDL cholesterol (OR 0.96, 95\% CI 0.93-1.00, $p=0.003$ ), and current smoking status (OR 3.07, 95\% CI 1.17-8.08, $p=0.023$ ) were significant independent predictors for severe $\mathrm{CAD}$ in multivariate analysis (Table 5), which was in line with the less than CAC analyses. In this analysis, current smoking status was a consistent predictor for obstructive CAD (OR 3.82, 95\% CI 1.95-7.49, $p<0.001$ ) and severe CAD. However, low HDL cholesterol was a significant predictor for severe $\mathrm{CAD}$, while it was not for obstructive

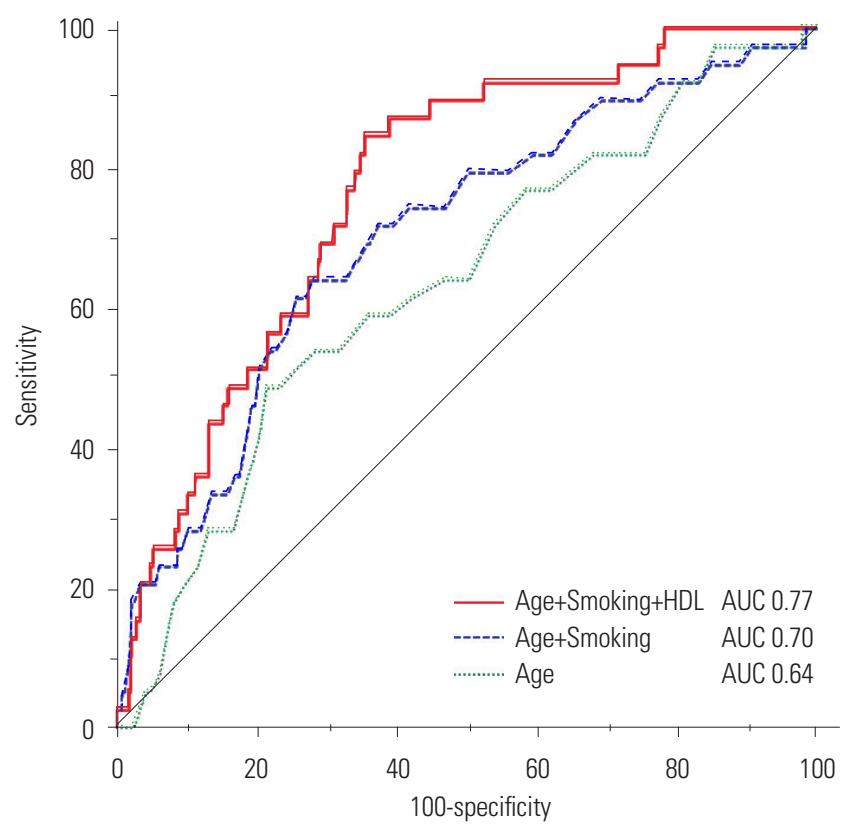

Fig. 2. Comparison of $\mathrm{C}$-statistics among the "Age+Smoking+HDL cholesterol" vs. "Age+Smoking" vs. "Age" model for predicting severe CAD. HDL, high density lipoprotein; CAD, coronary artery disease; AUC, area under the receiver operating characteristic curve.
CAD. The suggested cut-off value of low HDL cholesterol level to predict the presence of severe CAD was $46 \mathrm{mg} / \mathrm{dL}$, with a sensitivity of $74.4 \%$ and a specificity of $61.9 \%$.

\section{DISCUSSION}

Individuals with extremely low or even zero CAC are usually considered as free of significant CAD, especially those who are asymptomatic. Infrequently, however, serious $\mathrm{CAD}$ is observed in this population, which is a concern since a false negative study might cause optimal treatment times to be missed. In this study, we sought to find any clinical clues for prediction of such serious CAD in these falsely diagnosed populations by evaluating clinical CAD risk factors to identify any additional associations with moderate or severe CAD by CTA. We found that extremely small calcification, such as a CAC score of 1 to 9 , itself was associated with moderate $\mathrm{CAD}$, but not independently associated with severe $\mathrm{CAD}$, compared with zero CAC. Age, current smoking status, and extremely small calcification (CAC 1-9) were independently associated with moderate CAD. In addition, age, low HDL cholesterol, and current smoking status were shown to be independently associated with severe $\mathrm{CAD}$. We also discovered that current smoking status was a significant predictor of moderate and severe $\mathrm{CAD}$ among the less than $10 \mathrm{CAC}$ and even in the zero CAC population. Interestingly enough, low HDL cholesterol was only a significant predictor of severe $\mathrm{CAD}$, not moderate $\mathrm{CAD}$, among the less than 10 CAC or even in the zero CAC group. Moreover, a cut-off value of $46 \mathrm{mg} / \mathrm{dL}$ of HDL cholesterol showed a sensitivity of $74.4 \%$ and specificity of $61.9 \%$ for the prediction of severe CAD.

Coronary artery calcification indicates the presence of active inflammation or the stabilization of atherosclerotic plaque, and the total amount of coronary artery calcification is strongly

Table 4. Univariate and Multivariate Analysis for Detecting Moderate ( $\geq 50 \%$ Stenosis) CAD in the Zero CAC Population

\begin{tabular}{|c|c|c|c|c|c|c|}
\hline & \multicolumn{6}{|c|}{$C A C=0(n=972)$} \\
\hline & \multicolumn{3}{|c|}{ Univariate } & \multicolumn{3}{|c|}{ Multivariate } \\
\hline & Moderate CAD (odds ratio) & $95 \% \mathrm{CI}$ & $p$ value & Moderate CAD (odds ratio) & $95 \% \mathrm{Cl}$ & $p$ value \\
\hline Age & 1.04 & $1.02-1.07$ & $<0.001$ & 1.06 & $1.03-1.09$ & $<0.001$ \\
\hline Male & 1.86 & $1.08-3.20$ & 0.023 & 1.68 & $0.84-3.37$ & 0.15 \\
\hline $\mathrm{BMl}$ & 0.99 & $0.92-1.07$ & 0.78 & - & - & - \\
\hline DM & 2.91 & $1.54-5.52$ & 0.001 & 1.84 & $0.91-3.73$ & 0.090 \\
\hline HTN & 1.71 & $1.00-2.93$ & 0.049 & 0.86 & $0.46-1.61$ & 0.63 \\
\hline Dyslipidemia & 0.63 & $0.35-1.12$ & 0.12 & - & - & - \\
\hline Statin use & 0.72 & $0.40-1.28$ & 0.26 & - & - & - \\
\hline LDL cholesterol & 1.00 & $0.99-1.01$ & 0.63 & - & - & - \\
\hline HDL cholesterol & 0.97 & $0.95-0.99$ & 0.011 & 0.99 & $0.97-1.01$ & 0.25 \\
\hline TG & 1.00 & $1.00-1.00$ & 0.79 & - & - & - \\
\hline Creatinine & 1.13 & $0.88-1.44$ & 0.33 & - & - & - \\
\hline Current smoking & 3.86 & $2.24-6.65$ & $<0.001$ & 3.82 & $1.95-7.49$ & $<0.001$ \\
\hline
\end{tabular}

$\mathrm{CAD}$, coronary artery disease; CAC, coronary artery calcium; Cl, confidence interval; BMI, body mass index; DM, diabetes mellitus; HTN, hypertension; LDL, low density lipoprotein; HDL, high density lipoprotein; TG, triglyceride. 
Table 5. Univariate and Multivariate Analysis for Detecting Severe ( $\geq 70 \%$ Stenosis) CAD in the Zero CAC Population

\begin{tabular}{|c|c|c|c|c|c|c|}
\hline & & & $\mathrm{CAC}=0$ & 972) & & \\
\hline & & & & Mul & iate & \\
\hline & Severe CAD (odds ratio) & $95 \% \mathrm{Cl}$ & $p$ value & Severe CAD (odds ratio) & $95 \% \mathrm{Cl}$ & $p$ value \\
\hline Age & 1.05 & $1.02-1.09$ & 0.004 & 1.06 & $1.02-1.10$ & 0.002 \\
\hline Male & 1.63 & $0.74-3.58$ & 0.22 & 1.43 & $0.53-3.88$ & 0.48 \\
\hline BMl & 0.95 & $0.85-1.07$ & 0.38 & - & - & - \\
\hline DM & 3.22 & 1.32-7.87 & 0.010 & 1.71 & $0.67-4.35$ & 0.26 \\
\hline HTN & 1.08 & $0.48-2.45$ & 0.85 & - & - & - \\
\hline Dyslipidemia & 0.38 & $0.14-1.01$ & 0.051 & - & - & - \\
\hline Statin use & 0.41 & $0.15-1.10$ & 0.077 & - & - & - \\
\hline LDL cholesterol & 1.00 & 0.99-1.01 & 0.85 & - & - & - \\
\hline HDL cholesterol & 0.95 & $0.92-0.98$ & 0.001 & 0.96 & $0.93-1.00$ & 0.028 \\
\hline TG & 1.00 & $1.00-1.00$ & 0.48 & - & - & - \\
\hline Creatinine & 1.15 & $0.85-1.55$ & 0.37 & - & - & - \\
\hline Current Smoking & 3.25 & $1.47-7.21$ & 0.004 & 3.07 & $1.17-8.08$ & 0.023 \\
\hline
\end{tabular}

$\mathrm{CAD}$, coronary artery disease; $\mathrm{CAC}$, coronary artery calcium; $\mathrm{Cl}$, confidence interval; BMI, body mass index; DM, diabetes mellitus; HTN, hypertension; LDL, low density lipoprotein; HDL, high density lipoprotein; TG, triglyceride.

related to the total coronary plaque burden. ${ }^{8-10}$ Accordingly, coronary calcium scan has been established as a robust noninvasive CAD screening method, especially in the primary prevention population. Recently, several studies have demonstrated that CAC is a far superior discriminatory marker than global cardiovascular clinical risk factors. ${ }^{11-13}$ Many studies have also shown that CAC is directly proportional to the severity of CAD. ${ }^{2,13}$ However, severe CAD has been found in a considerable number of patients with low CAC. ${ }^{4,5,14}$ The incidences of significant $C A D$ in patients with zero or very low $C A C$ have varied in previous reports. ${ }^{15}$ Gottlieb, et al. ${ }^{5}$ reported that $19 \%$ had obstructive CAD $(\geq 50 \%)$ among the symptomatic zero CAC population ( $\mathrm{n}=291)$. However, Rubinshtein, et al ${ }^{16}$ and Koulaouzidis, et al. ${ }^{15}$ reported incidences of $7 \%(\mathrm{n}=231)$ and $0.9 \%$ $(\mathrm{n}=447)$, respectively, within the same symptomatic zero CAC population. As for the asymptomatic population, Choi, et al. ${ }^{4}$ and Lee, et al. ${ }^{17}$ reported incidences of $5 \%(\mathrm{n}=1000)$ and $0.7 \%$ ( $\mathrm{n}=6531$ ), respectively. In our study, obstructive CAD and severe $\mathrm{CAD}$ were identified in $6.0 \%$ and $2.7 \%$ of 972 asymptomatic subjects with zero CAC, respectively. In subjects with a less than 10 CAC score ( $\mathrm{n}=1176$ ), $7.5 \%$ were found to have obstructive CAD and 3.3\% severe CAD. Hence, as with the symptomatic population, significant $\mathrm{CAD}$ cannot be ruled out in asymptomatic individuals with zero CAC.

We found that, among traditional CAD risk factors, current smoking status was the strongest risk factor for obstructive CAD and severe CAD patients, while low HDL cholesterol was the strongest predictor associated with severe CAD. Smoking is an established CAD risk factor. ${ }^{18}$ It is also known that smoking leads to a decreased HDL cholesterol level through the dysfunction of lipid transport enzymes. ${ }^{19}$ In this study, low HDL and smoking seemed to be related with and have an effect on the development of coronary atherosclerosis. In addition, smoking can cause endothelial dysfunction, enhance platelet ag- gregation, and impair fibrinolysis. ${ }^{18}$ These alterations may be responsible for the increased prevalence and severity of thrombotic cardiovascular events in cigarette smokers. ${ }^{20}$

There are several limitations to our study. First, the number of patients was relatively small. In addition, it was limited to a single center study. It is necessary to follow up with a larger number of patients from a multi-center study to confirm our results. Second, the study was a retrospective study. Thus, similar to other retrospective studies, self-referral bias was a significant limitation. Third, various ethnicities were not considered in this study. Finally, plaque characteristics and prognostic information were not included in this analysis due to its crosssectional study design. Long-term follow up of prognoses with plaque characteristics might be needed to ascertain further clinical implications.

In conclusion, our analysis demonstrated that smoking is most strongly correlated with obstructive or severe $\mathrm{CAD}$, even in asymptomatic individuals with extremely low CAC scores. Low HDL cholesterol levels were also found to be a powerful predictor of severe CAD in the same subjects. These factors might be useful indicators worthy of consideration when screening for CAD in asymptomatic people.

\section{AUTHOR CONTRIBUTIONS}

Conceptualization: HBP, HJJ, YHC, and DKC. Data curation: HBP, HJJ, JHL, YSS, ESH, YHC, and DKC. Formal analysis: HBP, HJJ, and DKC. Funding acquisition: DKC. Investigation: HBP, HJJ, JHL, YSS, ESH, YHC, and DKC. Methodology: HBP, HJJ, JHL, YSS, ESH, YHC, and DKC. Project administration: HBP, HJJ, JHL, YHC, and DKC. Resources: HBP, HJJ, JHL, YSS, ESH, YHC, and DKC. Software: HBP, HJJ, and DKC. Supervision: HBP, HJJ, YHC, and DKC. Validation: HBP, HJJ, JHL, YSS, ESH, YHC, and DKC. Visualization: HBP and HJJ. Writingoriginal draft: HBP, HJJ, and DKC. Writing-review \& editing: HBP, HJJ, JHL, YSS, ESH, YHC, and DKC. 


\section{ORCID iDs}

Hyung-Bok Park Hyeonju Jeong Ji Hyun Lee Yongsung Suh Eui-Seock Hwang Yun-Hyeong Cho Deok-Kyu Cho https://orcid.org/0000-0002-3773-2665 https://orcid.org/0000-0002-7916-3624 https://orcid.org/0000-0003-1831-6735 https://orcid.org/0000-0002-1975-123X https://orcid.org/0000-0002-3025-8921 https://orcid.org/0000-0001-7581-9545 https://orcid.org/0000-0002-3881-411X

\section{REFERENCES}

1. Hecht HS. Coronary artery calcium scanning: past, present, and future. JACC Cardiovasc Imaging 2015;8:579-96.

2. Iwasaki K, Matsumoto T. Relationship between coronary calcium score and high-risk plaque/significant stenosis. World J Cardiol 2016;8:481-7.

3. Alluri K, Joshi PH, Henry TS, Blumenthal RS, Nasir K, Blaha MJ. Scoring of coronary artery calcium scans: history, assumptions, current limitations, and future directions. Atherosclerosis 2015; 239:109-17.

4. Choi EK, Choi SI, Rivera JJ, Nasir K, Chang SA, Chun EJ, et al. Coronary computed tomography angiography as a screening tool for the detection of occult coronary artery disease in asymptomatic individuals. J Am Coll Cardiol 2008;52:357-65.

5. Gottlieb I, Miller JM, Arbab-Zadeh A, Dewey M, Clouse ME, Sara $\mathrm{L}$, et al. The absence of coronary calcification does not exclude obstructive coronary artery disease or the need for revascularization in patients referred for conventional coronary angiography. J Am Coll Cardiol 2010;55:627-34.

6. Agatston AS, Janowitz WR, Hildner FJ, Zusmer NR, Viamonte M Jr, Detrano R. Quantification of coronary artery calcium using ultrafast computed tomography. J Am Coll Cardiol 1990;15:827-32.

7. DeLong ER, DeLong DM, Clarke-Pearson DL. Comparing the areas under two or more correlated receiver operating characteristic curves: a nonparametric approach. Biometrics 1988;44:837-45.

8. Rumberger JA, Simons DB, Fitzpatrick LA, Sheedy PF, Schwartz RS. Coronary artery calcium area by electron-beam computed tomography and coronary atherosclerotic plaque area. A histopathologic correlative study. Circulation 1995;92:2157-62.

9. Sangiorgi G, Rumberger JA, Severson A, Edwards WD, Gregoire J, Fitzpatrick LA, et al. Arterial calcification and not lumen stenosis is highly correlated with atherosclerotic plaque burden in humans: a histologic study of 723 coronary artery segments using nondecalci- fying methodology. J Am Coll Cardiol 1998;31:126-33.

10. Libby P. Mechanisms of acute coronary syndromes and their implications for therapy. N Engl J Med 2013;368:2004-13.

11. Hayward RA, Krumholz HM, Zulman DM, Timbie JW, Vijan S. Optimizing statin treatment for primary prevention of coronary artery disease. Ann Intern Med 2010;152:69-77.

12. Yeboah J, McClelland RL, Polonsky TS, Burke GL, Sibley CT, O'Leary D, et al. Comparison of novel risk markers for improvement in cardiovascular risk assessment in intermediate-risk individuals. JAMA 2012;308:788-95.

13. Budoff MJ, Nasir K, McClelland RL, Detrano R, Wong N, Blumenthal RS, et al. Coronary calcium predicts events better with absolute calcium scores than age-sex-race/ethnicity percentiles: MESA (Multi-Ethnic Study of Atherosclerosis). J Am Coll Cardiol 2009;53: $345-52$.

14. Villines TC, Hulten EA, Shaw LJ, Goyal M, Dunning A, Achenbach S, et al. Prevalence and severity of coronary artery disease and adverse events among symptomatic patients with coronary artery calcification scores of zero undergoing coronary computed tomography angiography: results from the CONFIRM (Coronary CT Angiography Evaluation for Clinical Outcomes: An International Multicenter) registry. J Am Coll Cardiol 2011;58:2533-40.

15. Koulaouzidis G, Charisopoulou D, Jenkins PJ, Koulaouzidis A, McArthur T. Prevalence of noncalcified coronary plaque in patients with calcium score of 0: the silent enemy. Angiology 2013;64: 205-10.

16. Rubinshtein R, Gaspar T, Halon DA, Goldstein J, Peled N, Lewis BS. Prevalence and extent of obstructive coronary artery disease in patients with zero or low calcium score undergoing 64-slice cardiac multidetector computed tomography for evaluation of a chest pain syndrome. Am J Cardiol 2007;99:472-5.

17. Lee MS, Chun EJ, Kim KJ, Kim JA, Yoo JY, Choi SI. Asymptomatic subjects with zero coronary calcium score: coronary CT angiographic features of plaques in event-prone patients. Int J Cardiovasc Imaging 2013;29 Suppl 1:29-36.

18. Katsiki N, Papadopoulou SK, Fachantidou AI, Mikhailidis DP. Smoking and vascular risk: are all forms of smoking harmful to all types of vascular disease? Public Health 2013;127:435-41.

19. Chelland Campbell S, Moffatt RJ, Stamford BA. Smoking and smoking cessation- the relationship between cardiovascular disease and lipoprotein metabolism: a review. Atherosclerosis 2008; 201:225-35.

20. Leone A. Smoking, haemostatic factors, and cardiovascular risk. Curr Pharm Des 2007;13:1661-7. 